\title{
Friction stir welding of a carbon-doped CoCrFeNiMn high-entropy alloy
}

\author{
D. Shaysultanov ${ }^{\mathrm{a}}$, N. Stepanov ${ }^{\mathrm{a}}$, S. Malopheyev ${ }^{\mathrm{b}}$, I. Vysotskiy ${ }^{\mathrm{b}}$, V. Sanin ${ }^{\mathrm{c}}$, S. Mironov $^{\mathrm{b}}$, \\ R. Kaibyshev ${ }^{\mathrm{b}}$, G. Salishchev ${ }^{\mathrm{a}}$, S. Zherebtsov ${ }^{\mathrm{a}, *}$ \\ a Laboratory of Bulk Nanostructured Materials, Belgorod State University, Pobeda 85, Belgorod 308015, Russia \\ ${ }^{\mathrm{b}}$ Laboratory of Mechanical Properties of Nanostructured Materials and Superalloys, Belgorod State University, Pobeda 85, Belgorod 308015, Russia \\ ${ }^{\mathrm{c}}$ Merzhanov Institute of Structural Macrokinetics and Materials Science, Russian Academy of Sciences, Academician Osipyan str. 8, Chernogolovka, Moscow Region \\ 142432, Russia
}

\section{A R T I C L E I N F O}

\section{Keywords:}

High entropy alloys

Friction stir welding

Recrystallization

Carbides

Mechanical properties

\begin{abstract}
A B S T R A C T
Butt-joint seam of a high entropy alloy (HEA) of the CoCrFeNiMn system was successfully obtained by friction stir welding (FSW). The HEA was produced by self-propagating high-temperature synthesis. Along with the principal elements, a small amount ( 0.9 at.\%) of $\mathrm{C}$ was added to the alloy. The as-cast alloy was cold rolled and annealed at $900{ }^{\circ} \mathrm{C}$ to produce refined microstructure. The structure of the annealed alloy consisted of a recrystallized face-centered cubic matrix with a grain size of $9.2 \mu \mathrm{m}$ and fine Cr-rich $\mathrm{M}_{23} \mathrm{C}_{6}$ carbides. FSW of the HEA resulted in microstructure refinement to $\mathrm{d}=4.6 \mu \mathrm{m}$ in the stir zone. A noticeable rise in strength and some decrease in ductility of the processed alloy in comparison with the initial condition can be associated with the microstructure refinement and some increase in the volume fraction of $\mathrm{M}_{23} \mathrm{C}_{6}$ carbides.
\end{abstract}

\section{Introduction}

High-entropy alloys (HEAs) are a new class of materials which usually consist of five (or more) metallic elements in nearly equimolar proportions [1]. Due to high entropy of mixing HEAs were supposed to crystallize as a solid solution phase(s), however the influence of high mixing entropy on the phase formation is in fact not so firm and many HEAs have two or more phases [2,3]. Nevertheless some HEAs demonstrate outstanding mechanical properties (high ductility under ambient conditions which becomes even higher with decreasing temperature and record-breaking fracture toughness at cryogenic temperature [4-6]) which attract growing interest to these materials. One of the promising classes of the HEAs is face-centered cubic (fcc) structured Co-Cr-Fe-Ni-Mn system alloys.

Although single fcc phase alloys (one of the typical representatives is the equiatomic CoCrFeNiMn alloy, also known as the Cantor alloy) possess high ductility and toughness, their strength is generally too low for advanced applications [4,7]. Significant efforts have been devoted to strengthening of such alloys. It was found that the most effective way is precipitation strengthening. To produce strengthening precipitates, modifications of chemical composition (i.e. addition of such elements as $\mathrm{Al}, \mathrm{Ti}, \mathrm{C}$, etc.) and proper heat treatment of the alloys are required
[8-15]. As a result, alloys with the strength level of $\sim 1 \mathrm{GPa}$ and sufficient ductility can be produced [16]. Among potential alloying elements, the interstitials like $\mathrm{C}$ or $\mathrm{N}$ attract special attention as they also (i) result in a considerable solid solution strengthening effect and (ii) can be used to change the predominance deformation mechanism due to a strong influence on stacking fault energy (SFE) of the fcc matrix [17-21].

Meanwhile a high level of mechanical properties is not the only important characteristic for a potential application of any structural material; the suitability of HEAs of various types to technological processing, such as welding, is also essential. Successful electron beam welding of the CoCrFeNiMn alloy without formation of solidification cracking was reported by Wu et al. [22]. Grain coarsening associated with very high heating temperature during electron-beam or laser-beam welding (as well as any other fusion welding techniques) might have an obvious negative effect on mechanical properties. However, fiber laser beam welding produced a defect-free butt joint of a CoCrFeNiMn-type HEA [23]. An increase in the microhardness in the fusion zone was attributed to the precipitation of nanoscale B2 particles, however such behavior is not expected to be typical of all HEAs.

To suppress grain coarsening (and even to refine microstructure) in the weld zone, a solid-state joining technique, for example friction stir

\footnotetext{
* Corresponding author.

E-mail addresses: shaysultanov@bsu.edu.ru (D. Shaysultanov), stepanov@bsu.edu.ru (N. Stepanov), malofeev@bsu.edu.ru (S. Malopheyev), visotsky@bsu.edu.ru (I. Vysotskiy), svn@ism.ac.ru (V. Sanin), rustam_kaibyshev@bsu.edu.ru (R. Kaibyshev), salishchev@bsu.edu.ru (G. Salishchev), zherebtsov@bsu.edu.ru (S. Zherebtsov).
} 
welding (FSW), can be used. In this method, a non-consumable rotating tool is inserted into the abutting edges of sheets or plates to be joined and traversed along the line of joint. Intensive friction heating softens the material around the tool thereby promoting a material motion from the front of the pin to the back of the pin [24]. Sound joints without welding defects were obtained by FSW of several HEAs belonging to the CoCrFeNi (Mn, Al) compositions [25-27]. The stir zone in all cases showed a refined equiaxed microstructure due to recrystallization. The hardness of the stir zone was found to be higher than that of the base metal due to the grain refinement in all cases [25-27]. Meanwhile tensile testing has shown that even a considerable microstructure refinement in the weld region (by a factor of $\sim 14$ when compared with the base metal) does not noticeably influence on strength of the CoCrFeNiMn HEA [27].

However, the effect of FSW on structure and mechanical properties of interstitial HEAs has never been studied. The presence of interstitial elements can potentially increase the strength of the joint due to intrinsic precipitation strengthening of the alloy during welding [23]. In the present work FSW of the CoCrFeNiMn alloy doped with $\mathrm{C}$ was studied. The alloy was produced by self-propagating high-temperature synthesis (SHS) which acquired a reputation as a suitable method for HEAs production [23,28].

\section{Materials and Methods}

The program CoCrFeNiMn alloy was produced using thermite-type SHS. A mixture of powders (oxides of the target elements (NiO, $\mathrm{Cr}_{2} \mathrm{O}_{3}$, $\mathrm{Co}_{3} \mathrm{O}_{4}, \mathrm{Fe}_{2} \mathrm{O}_{3}, \mathrm{MnO}_{2}$ ), pure carbon (C), and $\mathrm{Al}$ as the metal reducer) was used as a starting material. The synthesis of the program alloy was carried out in a graphite mold $80 \mathrm{~mm}$ in diameter using a centrifugal SHS setup. The obtained ingot was measured $80 \mathrm{~mm}$ in diameter $\times 15 \mathrm{~mm}$ in height.

The result of the chemical analysis (the concentrations of the metallic elements were measured by energy dispersive spectrometry (EDX) over area $\approx 1 \times 1 \mathrm{~mm}^{2}$, the concentration of carbon was measured using a LECO analyzer) of the obtained ingot is given in Table 1. Note that $\mathrm{Si}$ and $\mathrm{S}$ were found in the alloy that is most probably associated with impurity of the starting oxide powders.

Before FSW the HEA was cold rolled to $75 \%$ thickness reduction and then annealed at $900^{\circ} \mathrm{C}$ for $1 \mathrm{~h}$ to produce a uniform recrystallized microstructure. Coupons measured $40 \times 70 \times 2 \mathrm{~mm}^{3}$ were friction-stir butt welded perpendicular to rolling direction using an AccuStir 1004 FSW machine. In order to provide a full-penetration joining, a doubleside FSW was applied in the same direction. The welding process was performed in air using a WC-Co based tool with the shoulder diameter of $12.5 \mathrm{~mm}$ and a hemispherical pin of $1.5 \mathrm{~mm}$ in length. The tool rotation speed and the tool travel speed were kept at $1000 \mathrm{rpm}$ and $30 \mathrm{~mm} / \mathrm{min}$, respectively. The force applied on the rotating tool during FSW was $11.1 \mathrm{kN}$. Welding parameters were selected on the results of some preliminary trials to decrease the pin load on the one hand and to avoid too high temperature on the other hand. During FSW, the tool was tilted by $2.5^{\circ}$ from the sheet normal such that the rear of the tool was lower than the front.

For microstructural examinations, the produced weld was sectioned perpendicular to welding direction. Microstructural observations were performed by X-ray diffraction (XRD) analysis, scanning electron microscopy (SEM), electron backscatter diffraction (EBSD) technique and transmission electron microscopy (TEM). XRD analysis was performed using a RIGAKU diffractometer and $\mathrm{Cu} \mathrm{K} \alpha$ radiation. The final surface

Table 1

Measured chemical composition of the program alloy (in at.\%).

\begin{tabular}{lllllllll}
\hline Co & $\mathrm{Cr}$ & $\mathrm{Fe}$ & $\mathrm{Ni}$ & $\mathrm{Mn}$ & $\mathrm{Al}$ & $\mathrm{C}$ & $\mathrm{Si}$ & $\mathrm{S}$ \\
\hline 19.1 & 20.0 & 20.2 & 19.6 & 19.2 & 0.6 & 0.9 & 0.3 & 0.1 \\
\hline
\end{tabular}

finish for EBSD was obtained by polishing with an oxide polish suspension compound. The macrostructure of the FSW butt-joint was examined by SEM after etching in a solution of $50 \mathrm{ml}$ of $\mathrm{HCl}, 25 \mathrm{ml}$ of $\mathrm{H}_{2} \mathrm{SO}_{4}$ and $10 \mathrm{~g}$ of $\mathrm{CuSO}_{4}$ in $25 \mathrm{ml}$ of water. TEM specimens were produced by conventional twin-jet electro-polishing of mechanically pre-thinned to $100 \mu \mathrm{m}$ foils, in a mixture of $90 \% \mathrm{CH}_{3} \mathrm{COOH}$ and $10 \%$ $\mathrm{HClO}_{4}$ at the $27 \mathrm{~V}$ potential at room temperature. The EBSD analysis was conducted using an FEI Quanta 600 field-emission-gun scanning electron microscope (FEG-SEM) equipped with a TSL OIM ${ }^{\mathrm{TM}}$ software. In the EBSD maps high-angle boundaries (HABs) $\left(\geq 15^{\circ}\right)$ were depicted as black lines. The TEM observations were performed using a JEM-2100 TEM operating at $200 \mathrm{kV}$. At least 15 images taken from 3 different locations of foils were used to analyze the second phase(s) fraction.

Tensile tests at room temperature of dog-bone flat specimens (gage measured $6 \times 3 \times 1 \mathrm{~mm}^{3}$ ) were conducted at a constant crosshead velocity corresponding to a nominal strain rate of $10^{-3} \mathrm{~s}^{-1}$ using an Instron 5882 universal testing machine. The tensile specimens were cut both across and along the joint; in the latter case the gauge of the specimen entirely consisted of the friction-stir welded/processed material. Microhardness profiles across the joint were obtained using an automated Vickers hardness testing machine with a $0.3 \mathrm{~kg}$ load.

\section{Results}

The initial (cold rolled and recrystallized) condition (denoted further as the base material (BM)) of the alloy consisted of equiaxed grains with a size of $9.2 \pm 4.4 \mu \mathrm{m}$ (Fig. 1a, b). However almost every grain contained annealing twins which slightly decrease (to $\sim 7 \mu \mathrm{m}$ ) an average inter-boundary space. TEM images show small $\mathrm{M}_{23} \mathrm{C}_{6}$ carbide particles with a size of $138 \pm 47 \mathrm{~nm}$ within fcc matrix grains (Fig. 1c); the volume fraction of the carbides was $\sim 2 \%$. Due to low percentage of $\mathrm{M}_{23} \mathrm{C}_{6}$ phase the XRD pattern shows a single-phase fcc structure alloy without peaks corresponding to carbides (Fig. 1d).

Due to double-sided FSW the macrostructure of a transverse crosssection of the weld revealed a characteristic sand glass shape (Fig. 2). It should be noted that even at the etched surface the difference between the BM and the weld is hardly recognized; for the sake of simplicity the region of the transformed due to FSW structure is shown by dashed lines. As a result of double-sided FSW the retreating and advancing sides of the weld (RS and AS, respectively) are situated at the opposite sides of the workpiece at the top and the bottom sides. For the top side of the workpiece (as it is shown in Fig. 2) RS is on the left and AS is on the right side (and vice-versa for the bottom side). The weld contained no evident volumetric defects; moreover the boundary between the joint coupons cannot be recognized. However an obvious change of contrast (so-called white band (WB), indicated by arrows in Fig. 2) forming an irregular pattern can be seen at the AS of the stir zone (SZ).

EBSD maps obtained from different areas of the weld are shown in Fig. 3; these areas are indicated in Fig. 2 as $1-3$, respectively. The microstructure which approximately corresponds to the boundary between the joint coupons at the mid-thickness of the welded plates (Fig. 3a) consisted of fine grains with an average size of $2.1 \pm 1.3 \mu \mathrm{m}$. Some grains contained annealing twins. The shape of the grains was close to an equiaxed one, however grain boundaries were often found to be curved. Pronounced bulging of some boundaries was found (an example is shown with an arrow in at a high magnification insert in Fig. 3a). Also some coarser grains with a size of up to $7 \mu \mathrm{m}$ can be seen in the microstructure.

Area \#2 (Fig. 3b) corresponded to the SZ. In this area the microstructure was noticeably coarser $(d=4.6 \pm 2.4 \mu \mathrm{m})$. The number of grains which contain annealing twins was smaller in comparison with area \#1. It should be noted that grain boundaries were rather poorly determined most likely due to high internal stresses.

EBSD map from area \#3 shows a considerable variation in grain size (Fig. 3c). The smallest grain size of $2.0 \pm 0.9 \mu \mathrm{m}$ was observed at the outer bound of the WB (right bottom corner in Fig. 3d), however the 


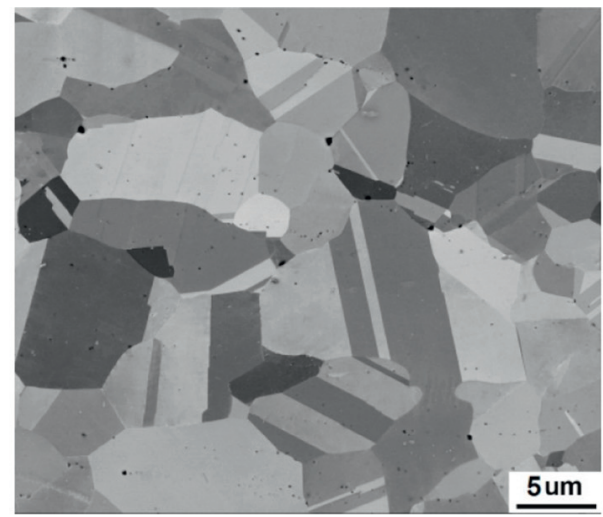

a

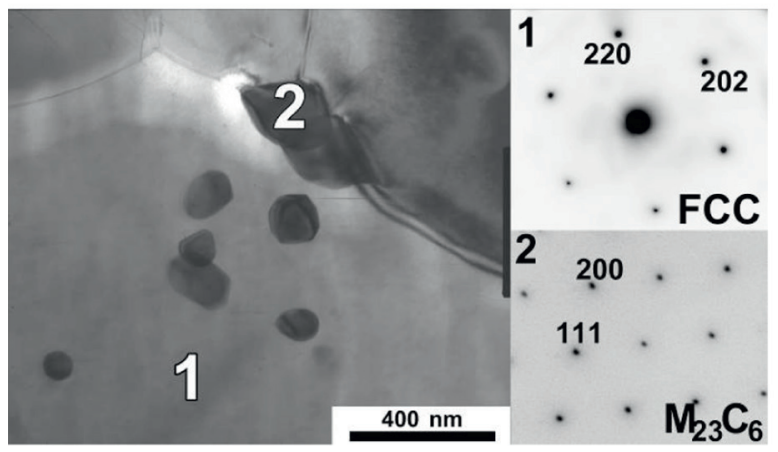

c

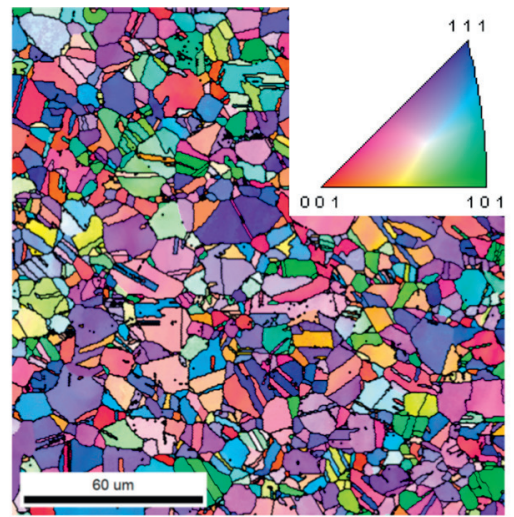

b

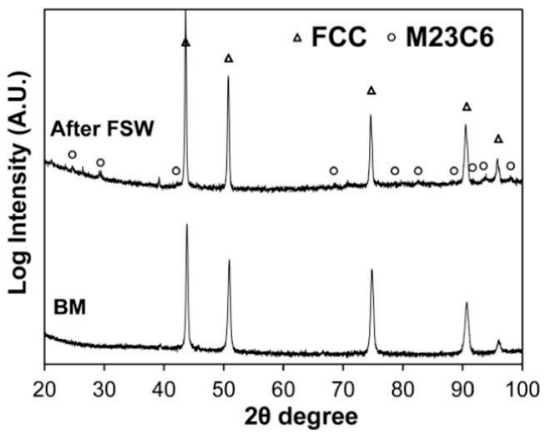

d

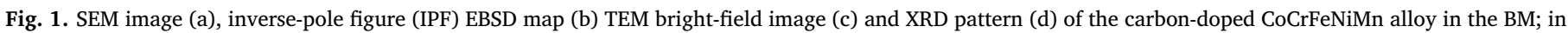

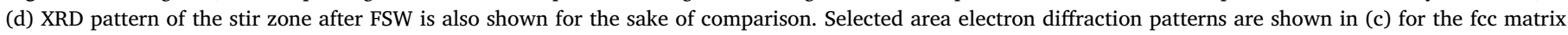
phase (1) and $\mathrm{M}_{23} \mathrm{C}_{6}$ carbides (2).

grain size approximately doubled at the inner bound of the WB (left top corner in Fig. 3d). Outside (but very close to) the WB the microstructure was noticeably coarser $(\mathrm{d} \approx 3.1 \pm 1.8 \mu \mathrm{m})$ (Fig. 3e). Some grains contained annealing twins.

Besides the smaller grain size, a closer examination of the WB microstructure showed the presence of rather small $(\mathrm{d}=0.5-2 \mu \mathrm{m})$ particles with a light contrast in SEM-BSE image (Fig. 4). The light contrast suggests that the particles were enriched with elements of high atomic number. Indeed, according to SEM-EDS analysis the particles contained $\sim 10$ at.\% of tungsten. These particles, rather homogeneously distributed in the microstructure, were not observed outside the WB. According to some previous works $[26,29]$ these particles can be associated with the wear of the WC-Co based tool during FSW.

Analysis of the misorientation distributions (Fig. 5) showed that the fraction of low-angle boundaries (LABs, $\leq 15^{\circ}$ ) fell in an interval of
$11-14 \%$ for all studied areas of the weld (Fig. 5 b-f); in the BM the fraction of LABs was just slightly greater (15,2\%) (Fig. 5a). In contrast the fraction of high-angle boundaries (HABs) was large enough providing quite a high average misorientation $\left(37.2^{\circ}-41.8^{\circ}\right.$ in the different areas of the weld and $43.2^{\circ}$ in the BM). Annealing twins with $\Sigma 3$ boundaries which corresponded to a peak at $60^{\circ}$ gave a considerable contribution into the fraction of HABs. The fraction of the twin boundaries was the highest (38\% among all boundaries) in BM. In all areas of the weld this peak also quite high (11-23\%), however a noticeable increase in the number of high-angle boundaries in an interval $30-60^{\circ}$ should also be noted (Fig. 5 b-f) in comparison to the BM (Fig. 5 a). It is also worth noting that the fraction of those boundaries which had low-to-medium misorientation $\left(10-20^{\circ}\right)$ was very low $(\leq 5.7 \%)$ in all studied areas, including the BM.

In the initial condition (cold rolling and then recrystallization), the

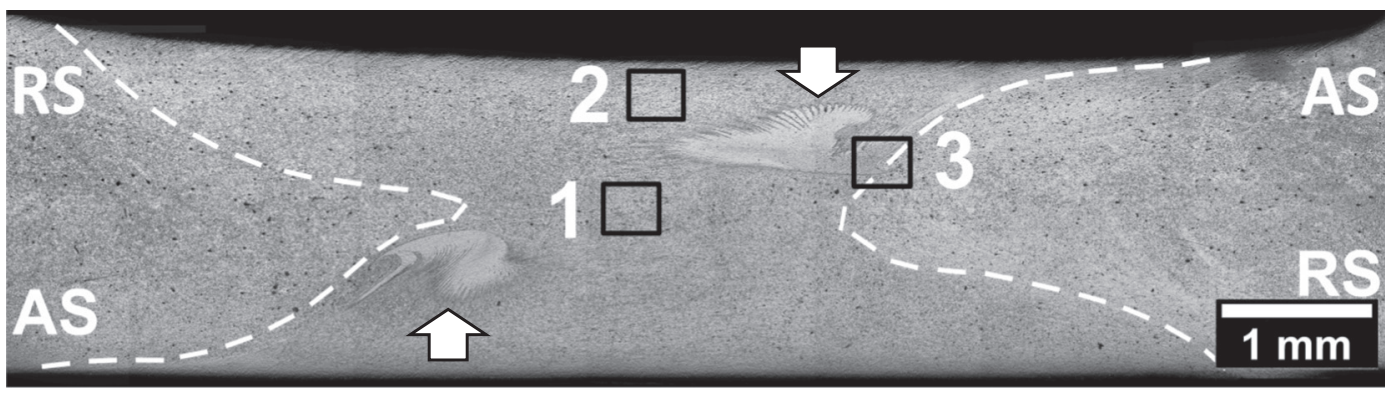

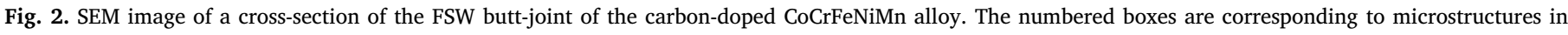
Fig. 3; the dashed lines show the weld boundaries; the arrows indicate the white bands (WBs). 


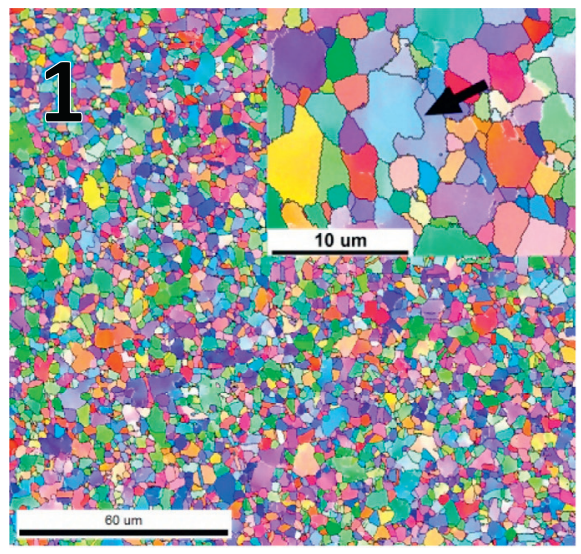

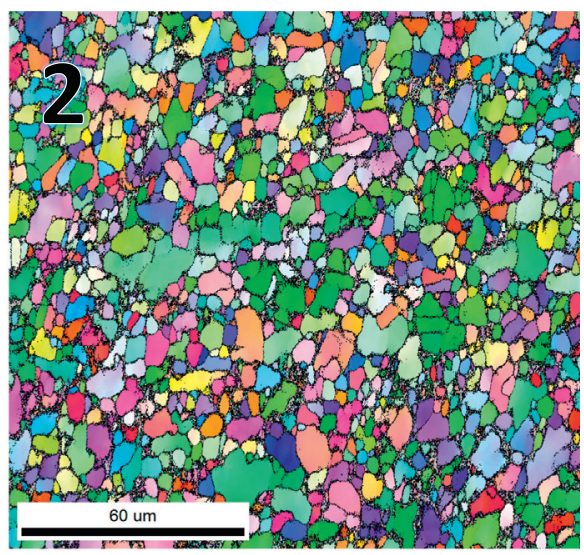

b

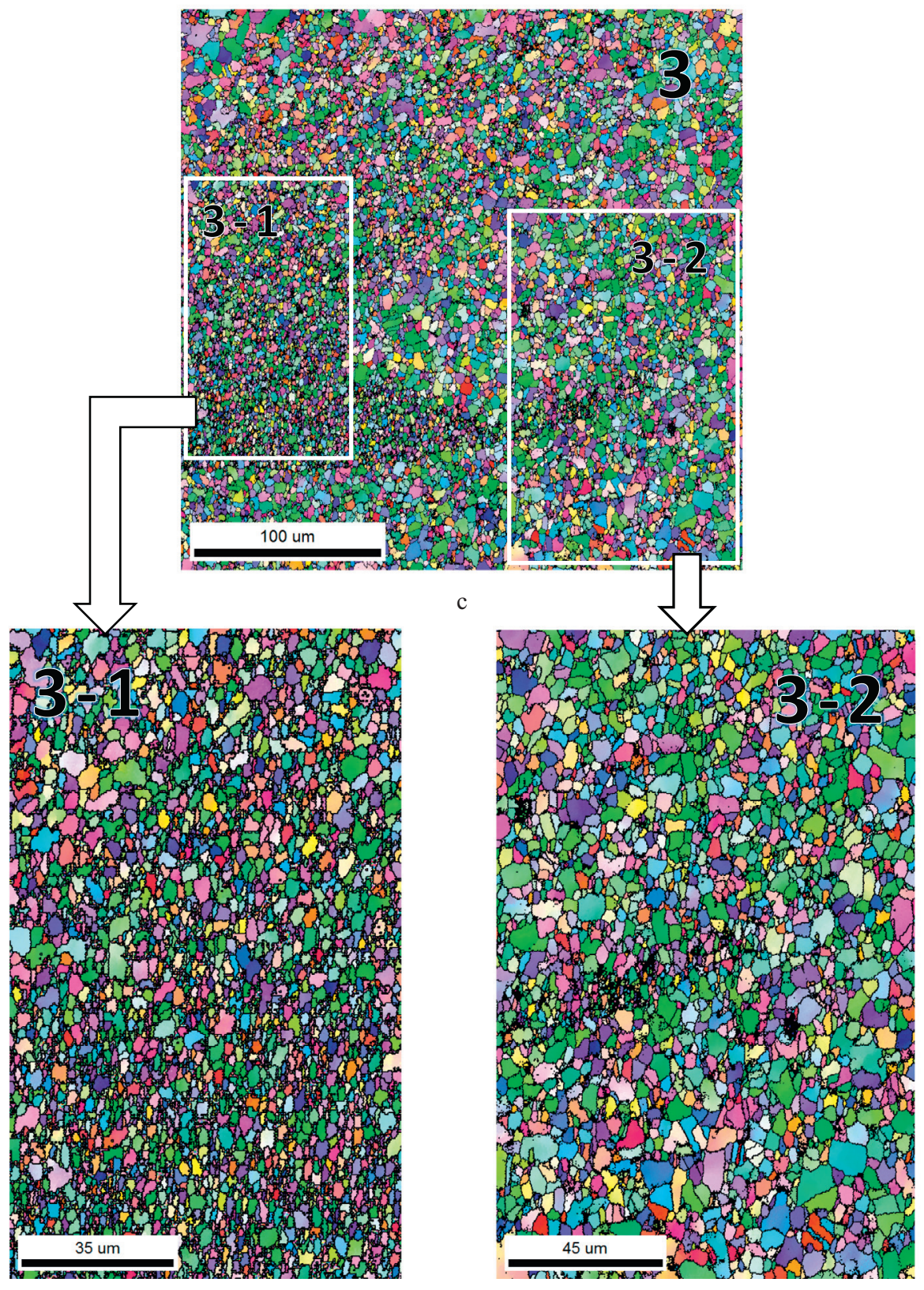




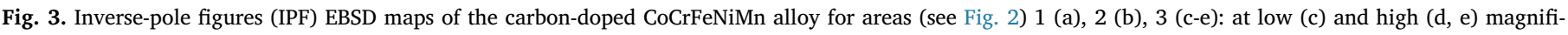
cations. The colour code for the IPF maps is shown in Fig. $1 \mathrm{~b}$.

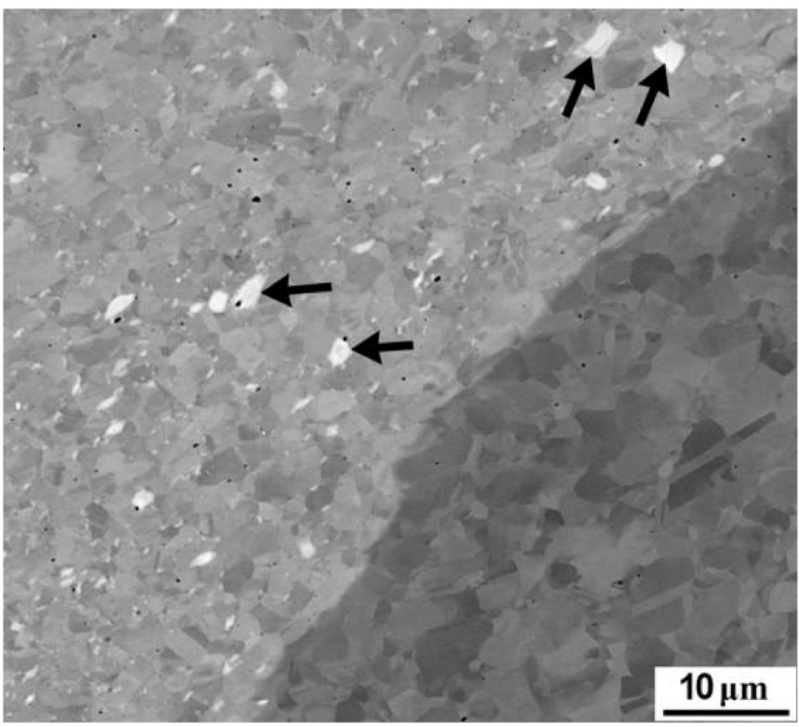

Fig. 4. SEM-BSE image of a border between the WB (light part) in the weld and the BM; few light particles contained $\mathrm{W}$ are marked with arrows.

material exhibited a weak irregular texture (Fig. 6a) which presumably originated from recrystallization [30] and the concomitant annealing twinning (Figs. 1a and b). Texture patterns after FSW changed significantly in comparison with the initial condition (Fig. 6). The overall texture (Fig. 6 b-d) was rather typical of the friction stir welded fcc alloys with relatively low stacking fault energy [30,31]. In areas \# 1 and 2 a texture of type $A_{1}$ (i.e. $\{111\}\langle\overline{1} 1 \overline{2}\rangle$ ) was formed (Fig. 6 b, c). It can be suggested that the material flow in the upper part of the weld (area \#2) was induced by the tool shoulder, whereas the texture formation in area \#1 was attributable to the probe tip [31]. The texture in area \#3 (the AS side of the SZ) can be ascribed to $A_{2}$ type (i.e. $\{111\}\langle 11 \overline{2}\rangle$ ) (Fig. 6d). In order to align the orientation data measured in this microstructural region with the presumed geometry of simple shear imposed during FSW [31], the experimental pole figures were rotated by $90^{\circ}$ around the normal direction and then tilted by $45^{\circ}$ around the transversal direction, as shown in Fig. $6 \mathrm{~d}$.

TEM (Fig. 7) of a specimen taken from the SZ (examined area roughly corresponded to \#2 in Fig. 3) shows a recrystallized microstructure with a very low dislocation density and clear, straight grain boundaries, often with extinction contrast (bottom part of Fig. 7). Similar to the $\mathrm{BM}$, the microstructure of the $\mathrm{SZ}$ contains $\mathrm{M}_{23} \mathrm{C}_{6}$ carbides, however some increase in the volume fraction and size (to $7 \%$ and $150 \pm 62 \mathrm{~nm}$, respectively) after FSW in comparison with that in the BM can be noted (see Fig. 1c). The majority of the carbides had sharp straight boundaries and a polygonal shape. Some increase in the fraction of $\mathrm{M}_{23} \mathrm{C}_{6}$ carbides is suggested by the result of XRD (Fig. 1d); in contrast to the $\mathrm{BM}$ for which XRD pattern shows a single-phase fcc structure, small peaks corresponding to $\mathrm{M}_{23} \mathrm{C}_{6}$ are observed in the XRD pattern for the SZ. The lower dislocation density and higher volume fraction of the carbides can be attributed to higher temperature attained during FSW (up to $1200^{\circ} \mathrm{C}$ [32]) in comparison with the temperature of recrystallization treatment $\left(900^{\circ} \mathrm{C}\right)$ during which the initial condition was obtained.

Microhardness measurement across the weld has shown a noticeable increase in hardness from $\sim 170 \mathrm{HV}$ in the BM to $\sim 210 \mathrm{HV}$ in the SZ (Fig. 8a). A peak value of the microhardness $(270 \mathrm{HV})$ was associated with the WB.

Tensile stress-strain curves of the carbon-doped CoCrFeNiMn alloy specimens in the initial condition and after FSW cut in different directions are shown in Fig. 8b,c. The main mechanical characteristics of the alloy in the initial condition and after FSW are also collected in Table 2. Due to the increased strength of the weld zone (YS $=490 \mathrm{MPa}$ against YS $=290-330 \mathrm{MPa}$ ), the produced joints have broken in the base material section during tensile tests of the specimens cut across the seam, as shown in the insert in Fig. 8b. The stress-strain curve shows, in comparison to the $\mathrm{BM}$, a decrease in ductility from $70 \%$ to $31 \%$ while the ultimate strength of both specimens was found to be rather close (UTS $=683 \mathrm{MPa}$ in the $\mathrm{BM}$ and $698 \mathrm{MPa}$ in the weld condition). However the decrease in ductility was an apparent effect associated with a much shorter gage due to the presence of a hard "insert" (the weld zone). Specimens cut along the weld show the maximum strength (UTS = $713 \mathrm{MPa}$ ) and high ductility of 55\% (Fig. 8c). Meanwhile the BM specimen cut across the rolling direction has noticeably lower strength (UTS $=630 \mathrm{MPa}$ ) both in comparison to the BM specimen cut along the rolling direction and the specimen cut along the seam (Fig. 8b, c). This phenomenon can be associated with i) some mechanical anisotropy which is proper for the cold rolled and recrystallized alloy (Fig. 6a) and ii) higher strength of the BM nearby the SZ most likely due to an increased fraction of the $\mathrm{M}_{23} \mathrm{C}_{6}$ carbides that induced by temperature rising during FSW. The latter can be seen from the microhardness distribution which shows hardened (in comparison to the $\mathrm{BM}$ ) area for $4-6 \mathrm{~mm}$ from the seam center.

\section{Discussion}

The use of FSW for a butt-jointing of carbon-doped CoCrFeNiMn HEA specimens resulted in the formation of a sound weld without any cracks or pores. Similar results with respect to $\mathrm{CoCrFeNiAl}{ }_{0.3}$ [25], CoCrFeNiMn [26], or $\mathrm{Co}_{16} \mathrm{Cr}_{28} \mathrm{Fe}_{28} \mathrm{Ni}_{28}$ [27] HEAs was reported earlier. Generally one of the obvious effects of FSW (or friction stir processing) is associated with considerable microstructure refinement due to severe deformation in the SZ and further recrystallization [33]. However the present alloy (unlike the previous compositions) contained carbide particles which are most probably responsible for the finer grain size in the initial condition in comparison with the "pure" CoCrFeNiMn alloy $(\sim 10 \mu \mathrm{m}$ vs $\sim 17 \mu \mathrm{m},[34])$. Therefore FSW in our case resulted just in a moderate microstructure refinement (to $4.6 \mu \mathrm{m}$ in the stir zone) (Figs. 1, 3b).

According to microstructure and misorientation distribution analysis (Figs. 3 and 5) the majority of grains have high-angle misorientation while the fraction of LABs was rather low that is typical of recrystallized condition. Considering very high temperature and large deformation in the SZ during FSW [24,33], dynamic recrystallization should inevitably occur during such a processing [35]. The lack of medium-angle boundaries and local bulging of some grain boundaries (Fig. 3a) suggest the prevalence of discontinuous mechanism of recrystallization which associated with migration of HABs (in contrast to continuous recrystallization when the new recrystallized grains originate from subgrains due to interaction of subboundaries with lattice dislocation and a gradual increase in LABs misorientation to the highangle condition) [36,37]. The presence of annealing twins in many grains and corresponding peaks at misorientation distributions in all areas (including the $\mathrm{BM}$ ) also suggests the development of discontinuous recrystallization [38].

In turn, a very low dislocation density in the SZ can rather be associated with static recrystallization because dynamic recrystallization usually reproduces a microstructure with quite a high dislocation density $[36,37]$. Therefore the microstructure evolution after FSW in the SZ was most likely associated with metadynamic recrystallization when recrystallization nuclei, appearing during dynamic 


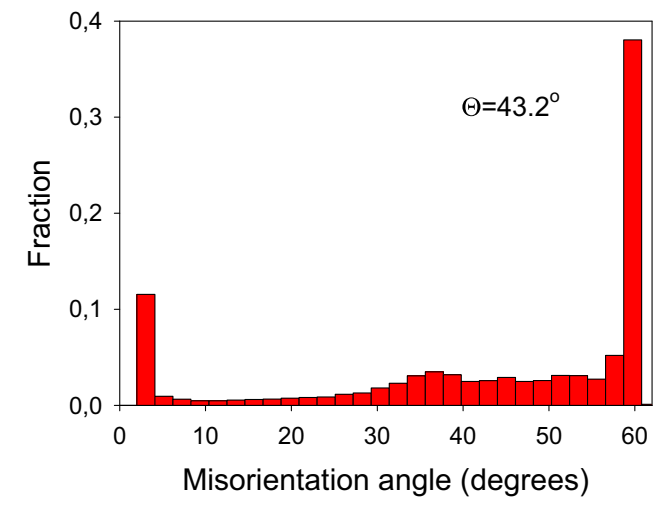

a

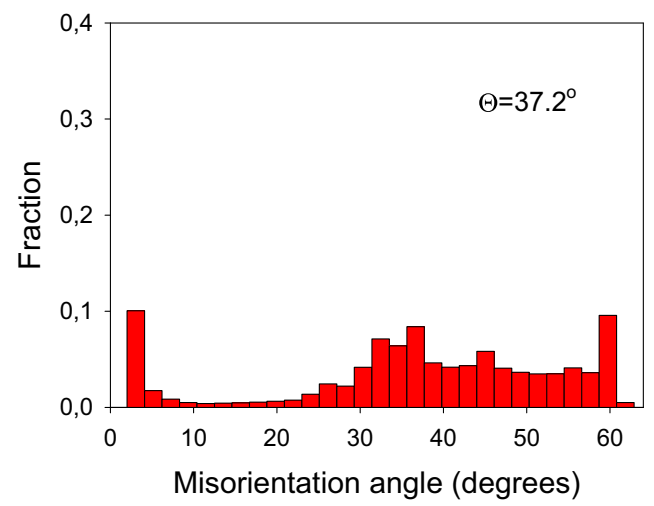

c

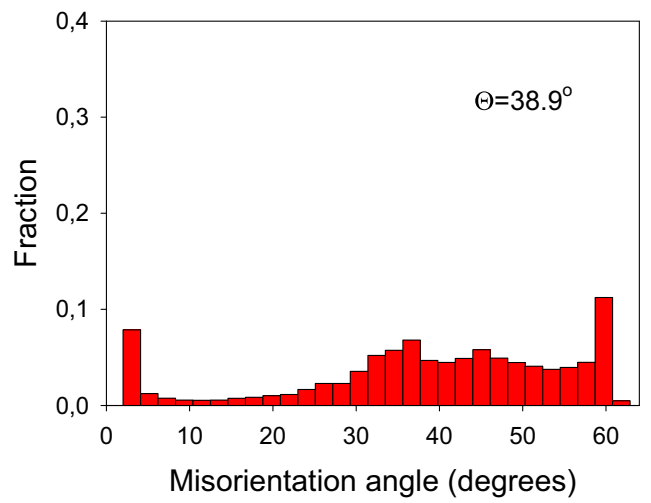

e

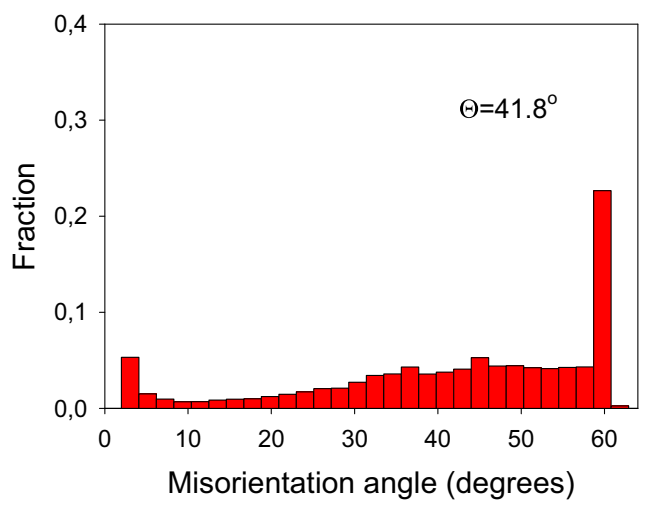

$\mathrm{b}$

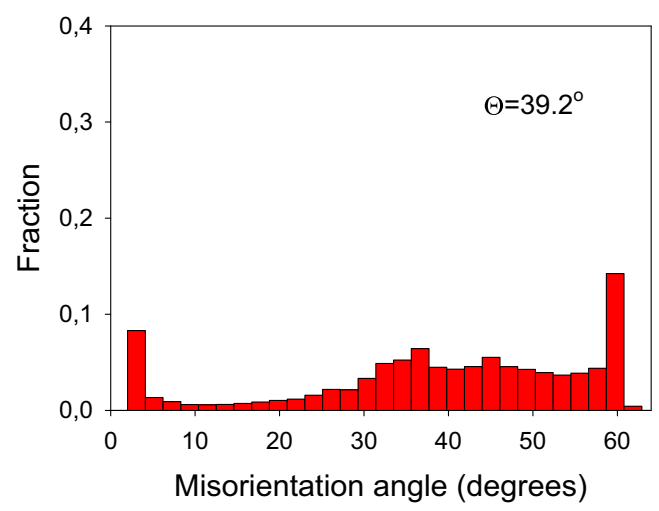

d

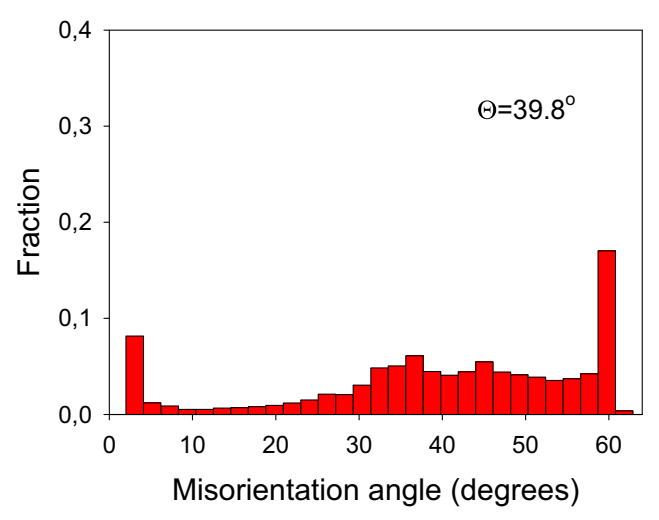

f

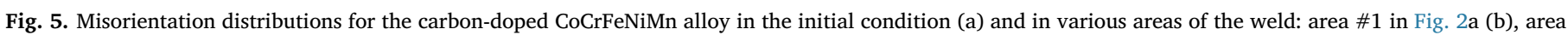
\#2 in Fig. 2a (c), area \#3 in Fig. 2a (d), area \#3-1 in Fig. 3a (e) and area \#3-2 in Fig. 3a (f).

recrystallization, grow with no incubation period into the dynamically recrystallized matrix [36].

The presence of tiny $(\sim 150 \mathrm{~nm}) \mathrm{M}_{23} \mathrm{C}_{6}$ carbides may have a limited influence on a well-known phenomenon associated with particle stimulated nucleation (PSN) of recrystallization. When the particles size is less than $\sim 1 \mu \mathrm{m}$ [36], dislocations at high temperatures may be able to bypass particles without forming deformation zones. However these carbide particles can limit nuclei growth thereby surviving rather fine and homogeneous microstructure. It worth noting that the $\mathrm{W}$-rich particles (see Fig. 4) can contribute in the PSN effect due to noticeably larger size (up to $2 \mu \mathrm{m}$ ) that in turn resulted in much finer microstructure $(\sim 2.0 \mu \mathrm{m})$ in area \#3-1 (Fig. 3d). Earlier noticeably finer microstructure in the WB in comparison to the rest of the SZ was also ascribed to the effect of PSN [26,39].

The formation of the annealing twins in all observed areas is also a marker of low staking fault energy (SFE) which is obviously inherent in the program material. The SFE for the equiatomic CoCrFeNiMn is known to be $20-30 \mathrm{~mJ} / \mathrm{m}^{-2}$ that is quite comparable to that of TWIP 

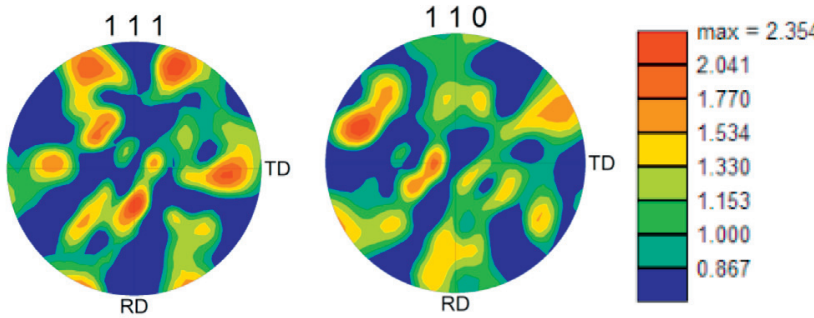

a

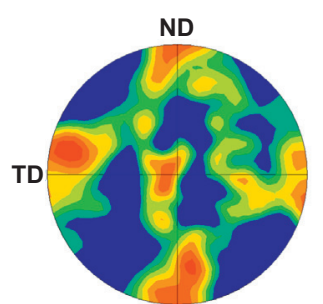

111

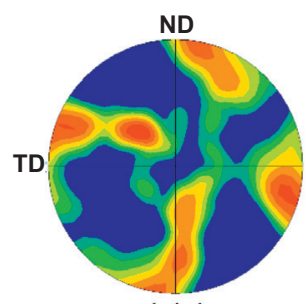

111

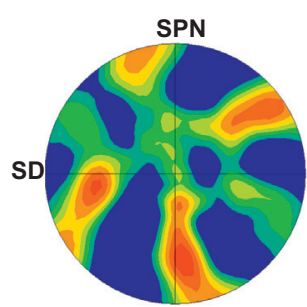

111

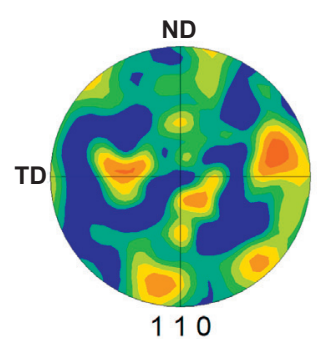

b

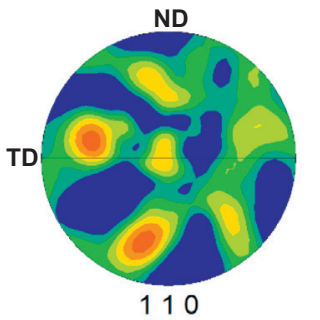

c

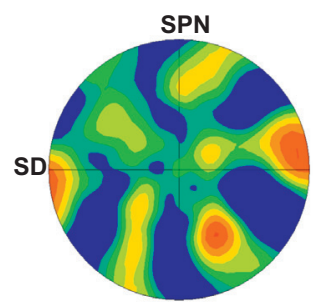

110

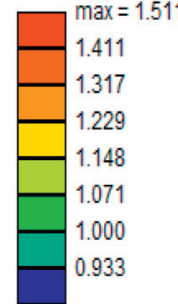

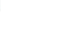



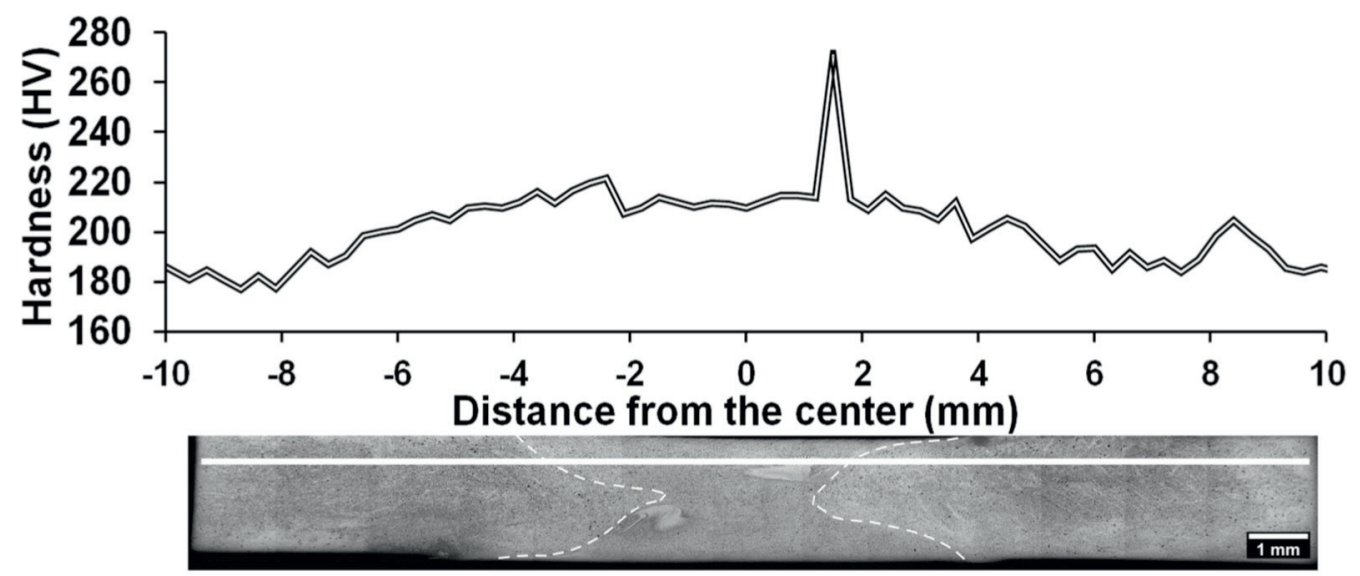

a

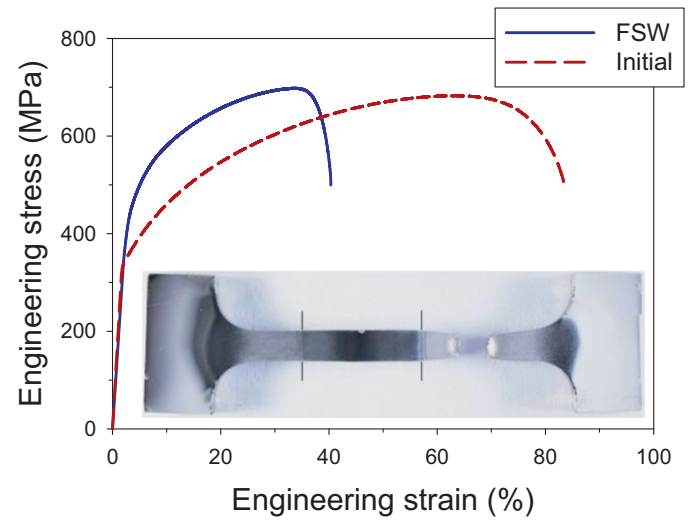

b

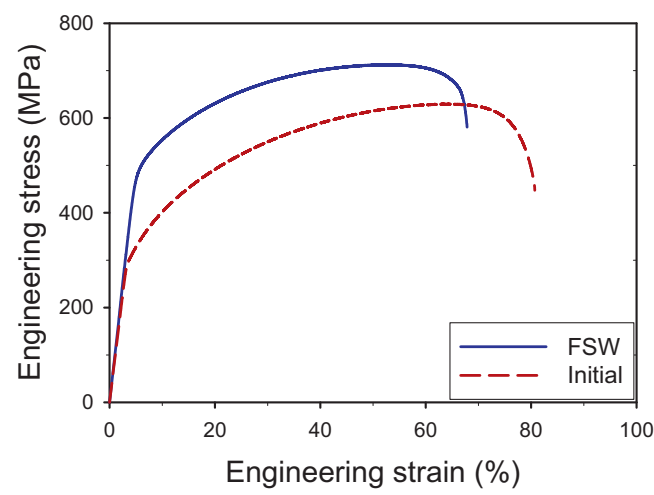

$\mathrm{c}$

Fig. 8. Microhardness distribution across the seam (a) and tensile stress-strain curves of the FSW specimens cut across (b) or along (c) the seam. The BM specimens (initial conditions) were cut parallel to the corresponding FSW specimens (along the RD in Fig. 8b and across the RD in Fig. 8c). A fractured tensile specimen is shown in Fig. 8b.

Table 2

Mechanical characteristics of the carbon-doped CoCrFeNiMn alloy in the initial condition and after FSW.

\begin{tabular}{lllll}
\hline Condition & Direction & YS, MPa & UTS, MPa & $\varepsilon, \%$ \\
\hline \multirow{2}{*}{ Initial } & Along the RD & 330 & 683 & 70 \\
\multirow{3}{*}{ FSW } & Across the RD & 290 & 630 & 65 \\
& Along the seam $(\perp$ to RD) & 490 & 713 & 52 \\
& Across the seam $(\|$ to RD) & 397 & 698 & 31 \\
\hline
\end{tabular}

joints are often considered as "weak" areas of fabricated structures even in the case of FSW [24,33]. Therefore the higher strength of the weld can potentially be an advantage for practical applications of the CoCrFeNiMn-type HEAs. An important fact is that no specific postwelding processing is needed to obtain a hardened joint, i.e. the alloy hardens due to the "natural" heat treatment during welding. Thus the present study shows one more advantage of interstitial alloying of HEAs. Moreover, it can be supposed that by tailoring microstructure via adjusting the chemical composition and processing parameters even higher strength of the FSW joints can be achieved in similar alloys $[11,23,47,48]$.

\section{Summary}

Successful friction stir welding of the carbon-doped CoCrFeNiMntype high entropy alloy was demonstrated. Sound welds without visible defects such as porosity or cracks were obtained. The difference in microstructure between the base material and welds was mainly associated with a decrease in grain size from $9.2 \mu \mathrm{m}$ in the BM to $4.6 \mu \mathrm{m}$ in the $\mathrm{SZ}$ and some increase in the volume fraction of the $\mathrm{M}_{23} \mathrm{C}_{6}$ carbide particles. These changes led to an increase in the ultimate tensile strength and yield strength by $\sim 80 \mathrm{MPa}$ and $200 \mathrm{MPa}$, respectively. The microhardness measurement also shows an approximately $40 \mathrm{HV}$ increase in the area of the SZ in comparison with the BM.

\section{Acknowledgments}

The authors gratefully acknowledge the financial support from the Russian Science Foundation grant no. 18-19-00003. The authors are grateful to the personnel of the Joint Research Center, "Technology and Materials", Belgorod State University, for their assistance with the instrumental analysis.

\section{References}

[1] J.W. Yeh, S.K. Chen, S.J. Lin, J.Y. Gan, T.S. Chin, T.T. Shun, C.H. Tsau, S.Y. Chang, Nanostructured high-entropy alloys with multiple principal elements: novel alloy design concepts and outcomes, Adv. Eng. Mater. 6 (2004) 299-303, https://doi. org/10.1002/adem.200300567.

[2] D.B. Miracle, O.N. Senkov, A critical review of high entropy alloys and related concepts, Acta Mater. 122 (2017) 448-511, https://doi.org/10.1016/j.actamat. 2016.08.081.

[3] Z.G. Zhu, K.H. Ma, Q. Wang, C.H. Shek, Compositional dependence of phase formation in three $\mathrm{CoCrFeNi}-(\mathrm{Mn} / \mathrm{Al} / \mathrm{Cu})$ high entropy alloys, Intermetallics 79 (2016) 1-11, https://doi.org/10.1016/j.intermet.2016.09.003.

[4] F. Otto, A. Dlouhý, C. Somsen, H. Bei, G. Eggeler, E.P. George, The influences of 
temperature and microstructure on the tensile properties of a CoCrFeMnNi highentropy alloy, Acta Mater. 61 (2013) 5743-5755, https://doi.org/10.1016/j. actamat.2013.06.018.

[5] B. Gludovatz, A. Hohenwarter, D. Catoor, E.H. Chang, E.P. George, R.O. Ritchie, A fracture-resistant high-entropy alloy for cryogenic applications, Science 345 (6201) (2014) 1153-1158, https://doi.org/10.1126/science.1254581.

[6] A. Gali, E.P. George, Tensile properties of high- and medium-entropy alloys, Intermetallics 39 (2013) 74-78, https://doi.org/10.1016/j.intermet.2013.03.018.

[7] A.J. Zaddach, R.O. Scattergood, C.C. Koch, Tensile properties of low-stacking fault energy high-entropy alloys, Mater. Sci. Eng. A 636 (2015) 373-378, https://doi. org/10.1016/j.msea.2015.03.109.

[8] J.Y. He, H. Wang, H.L. Huang, X.D. Xu, M.W. Chen, Y. Wu, X.J. Liu, T.G. Nieh K. An, Z.P. Lu, A precipitation-hardened high-entropy alloy with outstanding tensile properties, Acta Mater. 102 (2016) 187-196, https://doi.org/10.1016/j.actamat. 2015.08.076.

[9] J.Y. He, H. Wang, Y. Wu, X.J. Liu, H.H. Mao, T.G. Nieh, Z.P. Lu, Precipitation behavior and its effects on tensile properties of FeCoNiCr high-entropy alloys, Intermetallics 79 (2016) 41-52, https://doi.org/10.1016/j.intermet.2016.09.005.

[10] N.D. Stepanov, N.Y. Yurchenko, M.A. Tikhonovsky, G.A. Salishchev, Effect of carbon content and annealing on structure and hardness of the CoCrFeNiMn-based high entropy alloys, J. Alloys Compd. 687 (2016) 59-71, https://doi.org/10.1016/ j.jallcom.2016.06.103.

[11] N.D. Stepanov, D.G. Shaysultanov, R.S. Chernichenko, D.M. Ikornikov, V.N. Sanin, S.V. Zherebtsov, Mechanical properties of a new high entropy alloy with a duplex ultra-fine grained structure, Mater. Sci. Eng. A 728 (2018) 54-62, https://doi.org/ 10.1016/j.msea.2018.04.118.

[12] B. Gwalani, V. Soni, M. Lee, S. Mantri, Y. Ren, R. Banerjee, Optimizing the coupled effects of Hall-Petch and precipitation strengthening in a $\mathrm{Al}_{0.3} \mathrm{CoCrFeNi}$ high entropy alloy, Mater. Des. 121 (2017) 254-260, https://doi.org/10.1016/j.matdes. 2017.02.072.

[13] B. Gwalani, S. Gorsse, D. Choudhuri, M. Styles, Y. Zheng, R.S. Mishra, R. Banerjee, Modifying transformation pathways in high entropy alloys or complex concentrated alloys via thermo-mechanical processing, Acta Mater. 153 (2018) 169-185, https:// doi.org/10.1016/j.actamat.2018.05.009.

[14] K. Ming, X. Bi, J. Wang, Realizing strength-ductility combination of coarse-grained $\mathrm{Al}_{0.2} \mathrm{Co}_{1.5} \mathrm{CrFeNi}_{1.5} \mathrm{Ti}_{0.3}$ alloy via nano-sized, coherent precipitates, Int. J. Plast. 100 (2018) 177-191, https://doi.org/10.1016/j.ijplas.2017.10.005.

[15] Z.G. Zhu, Q.B. Nguyen, F.L. Ng, X.H. An, X.Z. Liao, P.K. Liaw, S.M.L. Nai, J. Wei, Hierarchical microstructure and strengthening mechanisms of a CoCrFeNiMn high entropy alloy manufactured by selective laser melting, Scr. Mater. 154 (2018) 20-24, https://doi.org/10.1016/j.scriptamat.2018.05.015.

[16] J.Y. He, H. Wang, H.L. Huang, X.D. Xu, M.W. Chen, Y. Wu, X.J. Liu, T.G. Nieh, K. An, Z.P. Lu, A precipitation-hardened high-entropy alloy with outstanding tensile properties, Acta Mater. 102 (2016) 187-196, https://doi.org/10.1016/j.actamat. 2015.08.076.

[17] Z. Wu, C.M. Parish, H. Bei, Nano-twin mediated plasticity in carbon-containing FeNiCoCrMn high entropy alloys, J. Alloys Compd. 647 (2015) 815-822, https:// doi.org/10.1016/j.jallcom.2015.05.224.

[18] N.D. Stepanov, D.G. Shaysultanov, R.S. Chernichenko, N.Y. Yurchenko, S.V. Zherebtsov, M.A. Tikhonovsky, G.A. Salishchev, Effect of thermomechanical processing on microstructure and mechanical properties of the carbon-containing CoCrFeNiMn high entropy alloy, J. Alloys Compd. 693 (2017) 394-405, https:// doi.org/10.1016/j.jallcom.2016.09.208.

[19] Z. Li, C.C. Tasan, H. Springer, B. Gault, D. Raabe, Interstitial atoms enable joint twinning and transformation induced plasticity in strong and ductile high-entropy alloys, Sci. Rep. 7 (2017) 40704, , https://doi.org/10.1038/srep40704.

[20] Z. Wang, I. Baker, Z. Cai, S. Chen, J.D. Poplawsky, W. Guo, The effect of interstitial carbon on the mechanical properties and dislocation substructure evolution in $\mathrm{Fe}_{40.4} \mathrm{Ni}_{11.3} \mathrm{Mn}_{34.8} \mathrm{Al}_{7.5} \mathrm{Cr}_{6}$ high entropy alloys, Acta Mater. 120 (2016) 228-239, https://doi.org/10.1016/j.actamat.2016.08.072.

[21] Z. Wang, I. Baker, W. Guo, J.D. Poplawsky, The effect of carbon on the microstructures, mechanical properties, and deformation mechanisms of thermo-mechanically treated $\mathrm{Fe}_{40.4} \mathrm{Ni}_{11.3} \mathrm{Mn}_{34.8} \mathrm{Al}_{7.5} \mathrm{Cr}_{6}$ high entropy alloys, Acta Mater. 126 (2017) 346-360, https://doi.org/10.1016/j.actamat.2016.12.074.

[22] Z. Wu, S.A. David, Z. Feng, H. Bei, Weldability of a high entropy CrMnFeCoNi alloy, Scr. Mater. 124 (2016) 81-85, https://doi.org/10.1016/j.scriptamat.2016.06.046.

[23] N. Kashaev, V. Ventzke, N. Stepanov, D. Shaysultanov, V. Sanin, S. Zherebtsov, Laser beam welding of a CoCrFeNiMn-type high entropy alloy produced by selfpropagating high-temperature synthesis, Intermetallics 96 (2018) 63-71, https:// doi.org/10.1016/j.intermet.2018.02.014.

[24] R.S. Mishra, Z.Y. Ma, Friction stir welding and processing, Mater. Sci. Eng. R 50 (2005) 1-78, https://doi.org/10.1016/j.mser.2005.07.001.

[25] Z.G. Zhu, Y.F. Sun, M.H. Goh, F.L. Ng, Q.B. Nguyen, H. Fujii, S.M.L. Nai, J. Wei, C.H. Shek, Friction stir welding of a $\mathrm{CoCrFeNiAl}_{0.3}$ high entropy alloy, Mater. Lett. 205 (2017) 142-144, https://doi.org/10.1016/j.matlet.2017.06.073.

[26] Z.G. Zhu, Y.F. Sun, F.L. Ng, M.H. Goh, P.K. Liaw, H. Fujii, Q.B. Nguyen, Y. Xu, C.H. Shek, S.M.L. Nai, J. Wei, Friction-stir welding of a ductile high entropy alloy: microstructural evolution and weld strength, Mater. Sci. Eng. A 711 (2018) 524-532, https://doi.org/10.1016/j.msea.2017.11.058.

[27] M.G. Jo, H.J. Kim, M. Kang, P.P. Madakashira, E.S. Park, J.Y. Suh, D.I. Kim, S.T. Hong, H.N. Han, Microstructure and mechanical properties of friction stir welded and laser welded high entropy alloy $\mathrm{CrMnFeCoNi}$, Met. Mater. Int. 24 (2018) 73-83, https://doi.org/10.1007/s12540-017-7248-x.

[28] V.N. Sanin, V.I. Yukhvid, D.M. Ikornikov, D.E. Andreev, N.V. Sachkova, M.I. Alymov, SHS metallurgy of high-entropy transition metal alloys, Dokl. Phys. Chem. 470 (2016) 145-149, https://doi.org/10.1134/S001250161610002X.

[29] K.H. Song, H. Fujii, K. Nakata, Effect of welding speed on the microstructural and mechanical properties of friction stir welded Inconel 600, Mater. Des. 30 (2009) 3972-3978, https://doi.org/10.1016/j.matdes.2009.05.033.

[30] R.W. Fonda, K.E. Knipling, Texture development in friction stir welds, Sci. Technol Weld. Join. 16 (2011) 288-294, https://doi.org/10.1179/1362171811Y. 0000000010

[31] S. Mironov, Y.S. Sato, H. Kokawa, H. Inoue, S. Tsuge, Structural response of superaustenitic stainless steel to friction stir welding, Acta Mater. 59 (2011) 5472-5481, https://doi.org/10.1016/j.actamat.2011.05.021.

[32] J.-H. Cho, D.E. Boyce, P.R. Dawson, Modelling of strain hardening during friction stir welding of stainless steel, Model. Simul. Mater. Sci. Eng. 15 (2007) 469-486, https://doi.org/10.1088/0965-0393/15/5/007.

[33] R. Nandan, T. DebRoy, H.K.D.H. Bhadeshia, Recent advances in friction-stir welding - process, weldment structure and properties, Prog. Mater. Sci. 53 (2008) 980-1023, https://doi.org/10.1016/j.pmatsci.2008.05.001.

[34] N.D. Stepanov, D.G. Shaysultanov, M.S. Ozerov, S.V. Zherebtsov, G.A. Salishchev, Second phase formation in the CoCrFeNiMn high entropy alloy after recrystallization annealing, Mater. Lett. 185 (2016) 1-4, https://doi.org/10.1016/j.matlet. 2016.08.088

[35] N.D. Stepanov, D.G. Shaysultanov, N.Yu. Yurchenko, S.V. Zherebtsov, A.N. Ladygin, G.A. Salishchev, M.A. Tikhonovsky, High temperature deformation behavior and dynamic recrystallization in CoCrFeNiMn high entropy alloy, Mater. Sci. Eng. A 636 (2015) 188-195, https://doi.org/10.1016/j.msea.2015.03.097.

[36] F. Humphreys, M. Hatherly, Recrystallization and Related Annealing Phenomena, second ed, Elsevier, Oxford, 2004.

[37] T. Sakai, A. Belyakov, R. Kaibyshev, H. Miura, J.J. Jonas, Dynamic and post-dynamic recrystallization under hot, cold and severe plastic deformation conditions, Prog. Mater. Sci. 60 (2014) 130-207, https://doi.org/10.1016/j.pmatsci.2013.09. 002

[38] M. Tikhonova, Y. Kuzminova, X. Fang, W. Wang, R. Kaibyshev, A. Belyakov, $\Sigma 3$ CSL boundary distributions in an austenitic stainless steel subjected to multidirectional forging followed by annealing, Philos. Mag. 94 (2014) 4181-4196, https://doi.org/ 10.1080/14786435.2014.982743.

[39] Y.F. Sun, H. Fujii, The effect of SiC particles on the microstructure and mechanica properties of friction stir welded pure copper joints, Mater. Sci. Eng. 528 (2011) 5470-5475, https://doi.org/10.1016/j.msea.2011.03.077.

[40] A. Saeed-Akbari, L. Mosecker, A. Schwedt, W. Bleck, Characterization and prediction of flow behavior in high-manganese twinning induced plasticity steels: part I. Mechanism maps and work-hardening behaviour, Metall. Mater. Trans. A 43 (2011) 1688-1704, https://doi.org/10.1007/s11661-011-0993-4.

[41] M. Klimova, N. Stepanov, D. Shaysultanov, R. Chernichenko, N. Yurchenko, V. Sanin, S. Zherebtsov, Microstructure and mechanical properties evolution of the $\mathrm{Al}$, C-containing CoCrFeNiMn-type high-entropy alloy during cold rolling, Materials vol. 11, (1) (2017) 53, https://doi.org/10.3390/ma11010053.

[42] Z. Li, C.C. Tasan, H. Springer, B. Gault, D. Raabe, Interstitial atoms enable joint twinning and transformation induced plasticity in strong and ductile high-entropy alloys, Sci. Rep. 7 (2017) 40704, , https://doi.org/10.1038/srep40704.

[43] T. Gladman, Precipitation hardening in metals, Mater. Sci. Technol. 15 (1999) 30-36, https://doi.org/10.1179/026708399773002782.

[44] Z. Wu, H. Bei, G.M. Pharr, E.P. George, Temperature dependence of the mechanical properties of equiatomic solid solution alloys with face-centered cubic crystal structures, Acta Mater. 81 (2014) 428-441, https://doi.org/10.1016/j.actamat. 2014.08.026.

[45] N. Stepanov, M. Tikhonovsky, N. Yurchenko, D. Zyabkin, M. Klimova, S. Zherebtsov, A. Efimov, G. Salishchev, Effect of cryo-deformation on structure and properties of CoCrFeNiMn high-entropy alloy, Intermetallics 59 (2015) 8-17, https://doi.org/10.1016/j.intermet.2014.12.004.

[46] Z. Yanushkevich, A. Mogucheva, M. Tikhonova, A. Belyakov, R. Kaibyshev, Structural strengthening of an austenitic stainless steel subjected to warm-to-hot working, Mater. Charact. 62 (2011) 432-437, https://doi.org/10.1016/j.matchar. 2011.02.005

[47] S.S. Nene, K. Liu, M. Frank, R.S. Mishra, R.E. Brennan, K.C. Cho, Z. Li, D. Raabe, Enhanced strength and ductility in a friction strir processing engineered dual phase high entropy alloy, Sci. Rep. 7 (2017) 16167, , https://doi.org/10.1038/s41598017-16509-9.

[48] S.S. Nene, M. Frank, R.S. Mishra, B.A. McWilliams, K.C. Cho, Extremely high strength and work hardening ability in a metastable high entropy alloy, Sci. Rep. 8 (2018) 9920, , https://doi.org/10.1038/s41598-018-28383-0. 